\title{
gLIOSSARCOMA DE TRONCO CEREBRAL EM PACIENTE PEDIÁTRICO: RELATO DE CASO*
}

\author{
Roger Klein Moreira ${ }^{1}$, Daniela Koppe ${ }^{1}$, Juliana Zignani ${ }^{1}$, Marlon Cesar Marconato ${ }^{2}$, \\ Marcelo Abreu ${ }^{3}$, Eduardo Pitrez ${ }^{4}$, Álvaro Porto Alegre Furtado ${ }^{5}$
}

Resumo Os autores relatam o caso de um paciente branco, de quatro anos de idade, com comprometimento neurológico progressivo. Tomografia computadorizada do crânio e ressonância magnética evidenciaram lesão expansiva no tronco cerebral. Subseqüentemente, foi feito diagnóstico histopatológico de gliossarcoma. Trata-se de um tumor raro do sistema nervoso central que, na grande maioria dos casos, acomete pacientes acima dos 40 anos de idade e tem localização supratentorial. Há poucos casos relatados de gliossarcomas em crianças, sobretudo na região infratentorial. Dados epidemiológicos, assim como achados mais freqüentes na tomografia computadorizada e ressonância magnética, são discutidos.

Unitermos: Gliossarcoma; Tumores cerebrais; Radiologia; Tomografia computadorizada; Imagem por ressonância magnética.

Abstract Brain stem gliosarcoma in pediatric patient: a case report.

The authors report a case of a white four-year-old boy with progressive neurological symptoms due to a brain stem mass lesion identified by computed tomography and magnetic resonance imaging scans. The diagnosis of gliosarcoma was confirmed by histological examination. This rare type of central nervous system tumor affects mainly patients over $\mathbf{4 0}$ years of age and is most commonly supratentorial. Only a few cases of gliosarcoma in children have been reported, particularly in the infratentorial region. The epidemiological data as well as the most frequent computed tomography and magnetic resonance imaging findings are discussed.

Key words: Gliosarcoma; Brain tumors; Radiology; Computed tomography; Magnetic resonance imaging.

\section{INTRODUÇÃO}

O gliossarcoma é um tumor do sistema nervoso central (SNC), composto de células gliais neoplásicas em associação com elementos sarcomatosos de células fusiformes.

Desde a sua descrição original, feita por Ströbe ${ }^{(1)}$, em 1895 , este tumor vem sendo estudado quanto às suas características histopatológicas. Atualmente, a Intenational Society of Neuropathology classifica os gliossarcomas como um subtipo de glioblastoma. Trata-se de um tumor raro, geralmente de localização supratentorial, que

* Trabalho realizado no Serviço de Radiologia do Hospital de Clínicas de Porto Alegre (HCPA), Porto Alegre, RS.

1. Doutorandos da Faculdade de Medicina da Universidade Federal do Rio Grande do Sul (UFRGS).

2. Doutorando da Faculdade de Medicina de Santa Maria (UFSM).

3. Médico Residente do Serviço de Radiologia do HCPA.

4. Médico Contratado do Serviço de Radiologia do HCPA.

5. Professor de Radiologia da UFRGS, Chefe do Serviço de Radiologia do HCPA.

Endereço para correspondência: Dr. Eduardo Pitrez. Rua Marco Polo, 505, ap. 814, Bairro Cristo Redentor. Porto Alegre, RS, 91350-280. E-mail: epitrez@dualnet.com.br

Recebido para publicação em 24/1/2003. Aceito, após revisão, em 6/5/2003. geralmente acomete idosos, sendo incomum em pacientes com menos de 40 anos de idade $^{(\mathbf{1}-\mathbf{3})}$.

Relatamos, neste trabalho, um caso raro de um paciente de quatro anos de idade com diagnóstico de gliossarcoma de tronco cerebral.

\section{RELATO DO CASO}

Paciente de quatro anos de idade, do sexo masculino, branco, previamente hígido, procurou atendimento com queixa de fraqueza e dor nos membros inferiores, associadas a episódios de vômito e cefaléia nas regiões frontal e occipital. O quadro vinha progredindo há cerca de quatro meses. Ao exame físico havia sinais de lesão no trato piramidal.

Foram realizadas tomografia computadorizada (TC) e ressonância magnética (RM) do crânio, que mostraram lesão expansiva com componente cístico na topografia de quarto ventrículo, ponte, bulbo e pedúnculo cerebral esquerdo, medindo cerca de $3 \mathrm{~cm}$ de diâmetro, além de hidrocefalia não-comunicante (Figuras 1 e 2).
Foi feita biópsia da lesão, que revelou glioma de alto grau, com componente sarcomatoso, sendo diagnosticado gliossarcoma de tronco cerebral. Biópsia de medula óssea não mostrou presença de neoplasia nos cortes examinados. Um estudo tomográfico não evidenciou metástases em órgãos torácicos ou abdominais.

Apesar de ter sido iniciada radioterapia, o paciente evoluiu com hipertensão intracraniana e acometimento neurológico motor progressivos.

Exame tomográfico realizado três meses após o diagnóstico evidenciou aumento das dimensões do tumor e progressão da hidrocefalia não-comunicante (Figura 3).

O paciente faleceu seis meses após o diagnóstico.

\section{DISCUSSÃO}

Gliossarcomas são tumores malignos primários do SNC que apresentam componentes neuroectodérmicos e mesenquimais. Ainda que não haja um consenso sobre o assunto, sugere-se que os gliossarcomas originem-se a partir da transformação 


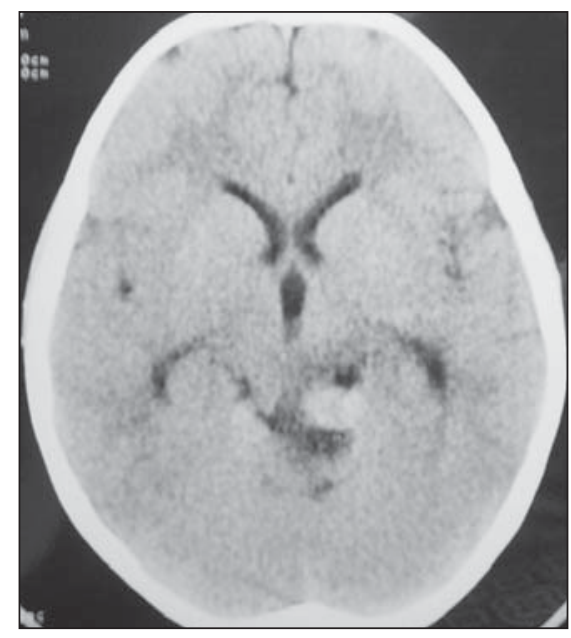

A

Figura 1. Tomografia computadorizada do crânio sem (A) e com contraste (B) evidenciando lesão hiperdensa na cisterna quadrigeminal e colículo superior à esquerda, apresentando impregnação ao contraste.

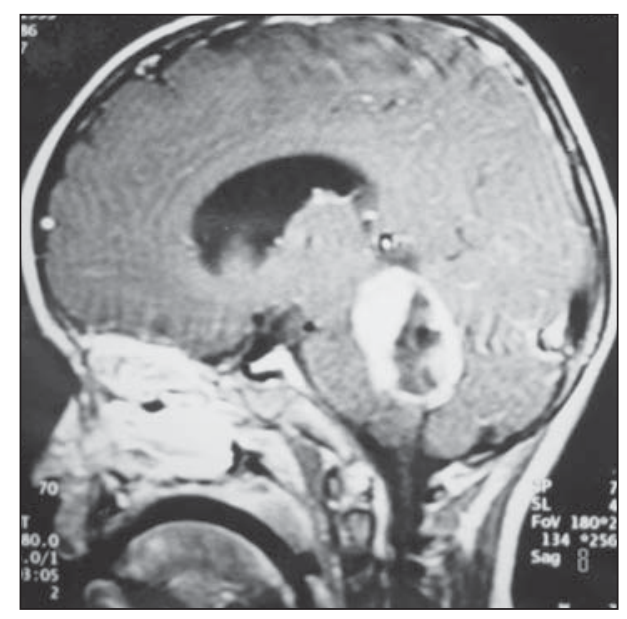

A

Figura 2. Ressonância magnética, planos axial (A) e sagital (B) ponderados em T1 após o contraste. Lesão bem delimitada comprometendo o quarto ventrículo e estruturas adjacentes com impregnação periférica ao contraste determinando leve hidrocefalia.

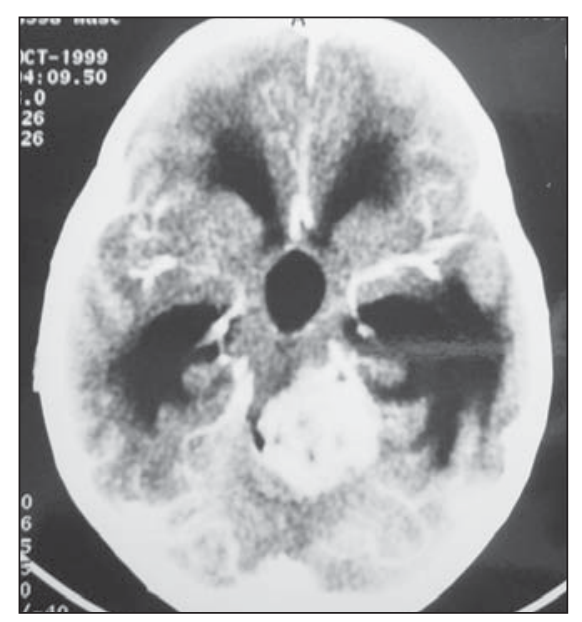

A

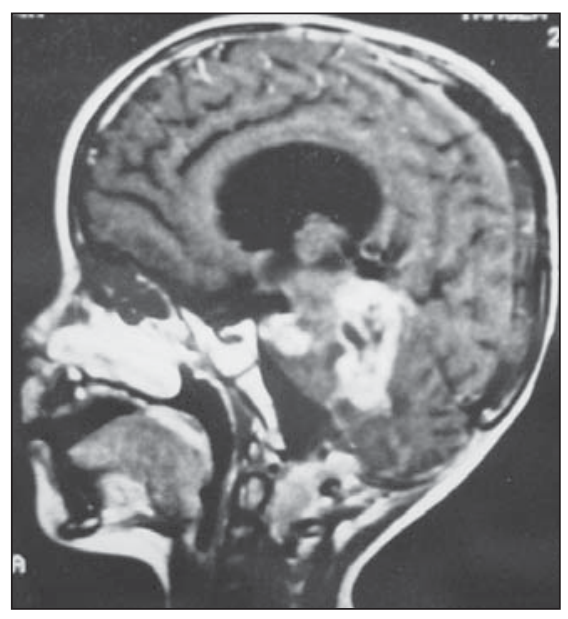

B

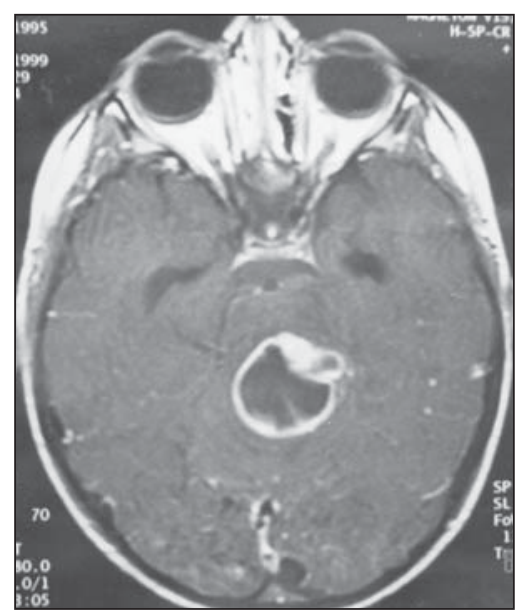

B

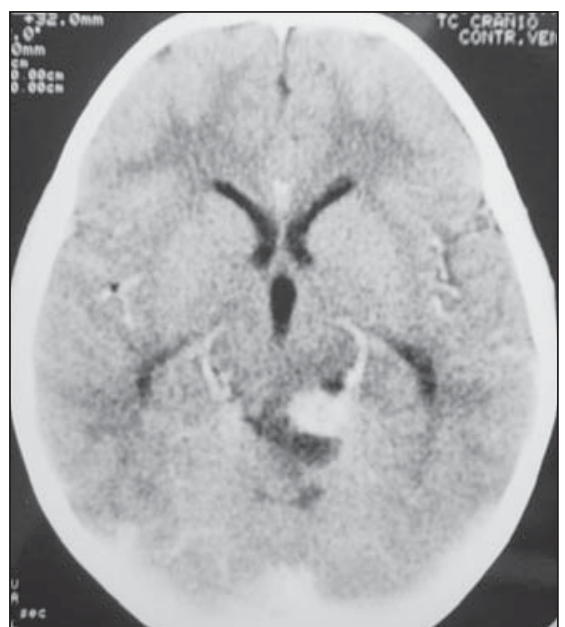

B neoplásica de elementos vasculares de um glioblastoma multiforme pré-existente ${ }^{(2)}$. A origem do componente sarcomatoso deste tumor, contudo, ainda gera grande controvérsia. Histologicamente, o componente glial destes tumores não difere daquele observado no glioblastoma multiforme. Acredita-se que a transformação sarcomatosa ocorra em menos de $2 \%$ dos glioblastomas multiformes ${ }^{(2,3)}$.

Segundo diversos estudos, na grande maioria dos casos os pacientes têm idades que variam de 40 a $70 \operatorname{anos}^{(\mathbf{1 - 3})}$. Apesar de existirem casos relatados de gliossarcomas em pacientes pediátricos, a maior parte dos trabalhos não reporta casos de pacientes jovens com este tipo de tumor ${ }^{(\mathbf{1 , 2})}$.

Há uma pronunciada tendência de este tipo de tumor ter localização supratentorial $^{(1-4)}$, mais especificamente nas regiões periféricas dos lobos temporais, geralmente tendo contato com o crânio ou com a foice cerebral ${ }^{(1,3)}$.

Alguns trabalhos afirmam que metástases sistêmicas de gliossarcomas são incomuns ${ }^{(2,5,6)}$. Outros trabalhos, entretanto, mostram que ocorrem metástases sistêmicas em até um terço dos casos, sendo possível, portanto, que este tumor metastatize com freqüência significativamente maior que o glioblastoma multiforme ${ }^{(\mathbf{1})}$.

Os achados tomográficos em casos de gliossarcoma são bastante variáveis, podendo ocorrer padrão semelhante ao observado em meningiomas, em alguns casos, ou semelhante ao dos glioblastomas multiformes, em outros. Áreas hiperdensas podem ser observadas na TC, provavelmente devido à alta celularidade e vascularização características do tumor ${ }^{(3,7,8)}$. Em casos de tumores de localização periférica, o fato de haver contato limitado com a dura-máter, edema peritumoral significativo e áreas de densidade heterogênea pode auxiliar no diagnóstico diferencial com meningioma. Em estudo tomográfico sem contraste, a maioria dos gliossarcomas apresenta-se como área heterogeneamente hiperdensa, tipicamente impregnando-se de modo homogêneo após injeção de contraste. Impregnação apenas em áreas periféricas da lesão também pode ocorrer ${ }^{(3)}$.

$\mathrm{Na}$ RM, os gliossarcomas costumam apresentar-se como lesões bem delimitadas, de aparência heterogênea ou cística, 
com edema vasogênico em sua periferia. Apesar de serem tumores intra-axiais, é freqüente que apresentem grande área de contato com a dura-máter. Em imagens ponderadas em T2, costumam apresentar intensidade de sinal intermediária, com sinais de edema periférico. A intensidade de sinal do tumor é semelhante àquela da substância cinzenta, menor do que a observada em outros tumores gliais. As imagens em T1 pós-contraste mostram impregnação intensa do tumor, freqüentemente mais pronunciada em áreas periféricas da lesão. Os gliossarcomas devem ser incluídos no diagnóstico diferencial de qualquer tumor de aspecto intra-axial, mas que tenha extenso contato com a dura-máter ou, ainda, que seja menos intenso em T2 do que se esperaria de um tumor glial $^{(\mathbf{9})}$.
Em resumo, o gliossarcoma é um tumor raro, resultante de transformação sarcomatosa que ocorre em uma minoria dos glioblastomas multiformes. Tradicionalmente considerado um tumor cerebral que acomete exclusivamente pacientes de meiaidade ou idosos, o gliossarcoma tem sido relatado, ainda que esporadicamente, em jovens e crianças. Torna-se necessário, portanto, que o gliossarcoma seja incluído no diagnóstico diferencial de lesões expansivas do SNC, tanto supratentoriais quanto infratentoriais.

\section{REFERÊNCIAS}

1. Morantz RA, Feigin I, Ransohoff J III. Clinical and pathological study of 24 cases of gliosarcoma. J Neurosurg 1976;45:398-408.

2. Classen J, Hoffmann W, Kortmann RD, et al. Gliosarcoma: case report and review of the litera- ture. Acta Oncol 1997;36:771-4.

3. Perry JR, Ang LC, Bilbao JM, Muller PJ. Clinicopathologic features of primary and postirradiation cerebral gliosarcoma. Cancer 1995;75:2910-8.

4. Jack CR Jr, Bhansali DT, Chason JL, et al. Angiographic features of gliosarcoma. AJNR 1987;8: 117-22.

5. Garret R. Glioblastoma and fibrosarcoma of the brain with extracranial metastases. Cancer 1958;11: 888-94.

6. Cerame MA, Guthikonda M, Kohli C. Extraneural metastasis in gliosarcoma: a case report and review of the literature. Neurosurgery 1985;17:413-8.

7. Lee YY, Castillo M, Nauert C, Moser RP. Computed tomography of gliosarcoma. AJNR 1985;6: 527-31.

8. Maiuri F, Stella L, Benvenuti D, Giamundo A, Pettinato G. Cerebral gliosarcomas: correlation of computed tomographic findings, surgical aspect, pathological features, and prognosis. Neurosurgery 1990;26:261-7.

9. Dwyer KW, Naul LG, Hise JH. Gliosarcoma: MR features. J Comput Assist Tomogr 1996;20:71923 